\title{
JÓVENES SIN EMPLEO, EMERGENCIA SOCIAL
}

\author{
Vicente Castelló Roselló \\ Profesor Universitat Jaume I de Castelló \\ y miembro del Instituto Interuniversitario de Desarrollo Local
}

\section{ÌNDICE}

1. Introducción

2. Desigualdades en el mercado laboral

3. Condiciones laborales más precarias

4. Dificultades en el acceso a la vivienda

5. Ante un problema sistémico, soluciones múltiples

5.1 Renovación sistema educativo

5.2 Impulsar Políticas Activas de Empleo

\section{INTRODUCCIÓN}

Las restricciones sanitarias impuestas por la pandemia del Covid-19 han provocado en el año 2020 una caída de proporciones históricas del producto interior bruto (PIB) y del empleo en las economías mundial y en la Unión Europea (UE) con gravísimas consecuencias sociales y económicas (gráfico 1). 


\section{El Producto Interior Bruto en la Unión Europea}

\section{CAÍDA DEL PIB EN 2020}

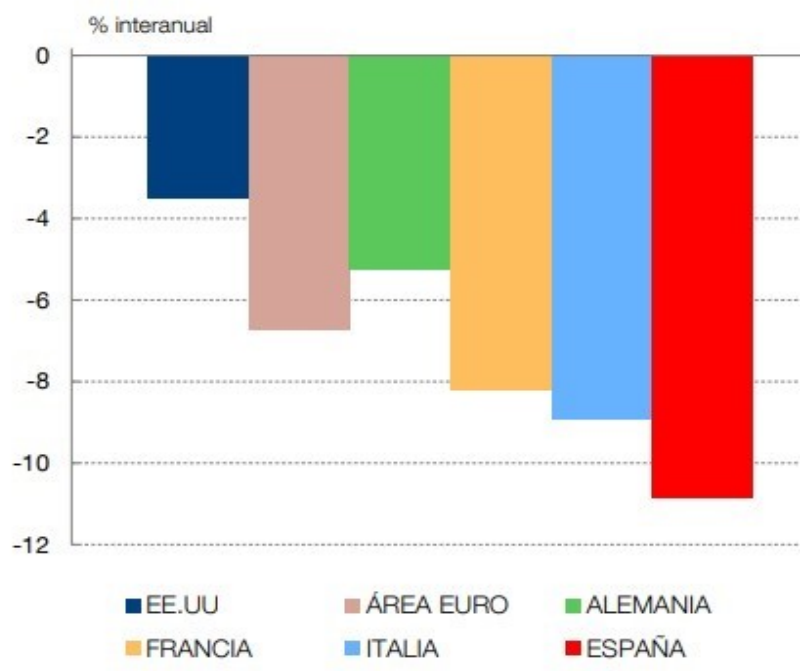

Fuente: Banco de España

Gráfico 1

España no ha sido ajena a todo ello y ha sufrido también un fuerte descenso de la actividad económica, que se ha traducido en un empeoramiento del mercado laboral, pero afectando de forma asimétrica entre los distintos grupos de edad. La destrucción de empleo se ha cebado en los más vulnerables, es decir, en aquellas personas que tienen un menor nivel de cualificaciones académicas $y$, 'por lo tanto, tienen una empleabilidad más reducida.

Como en todas las crisis económicas son los jóvenes los primeros perdedores, sobre todo los menos cualificados. Precisamente los sectores afectados por la pandemia emplean a un gran número de trabajadores jóvenes de poca formación que ahora requerirán una nueva adaptación.

En este contexto, el desempleo juvenil en España es un fenómeno estructural que ha persistido durante las últimas décadas, y se ha agravado durante la crisis sanitaria y económica derivada por el COVID-19. Muchos de los retos de la economía española anteriores a la pandemia, que con frecuencia se han visto agudizados por esta, afectan de manera especial a los jóvenes: sistema educativo, mercado laboral, vivienda, incertidumbre e inestabilidad de la renta.

Sus efectos han sido mucho más intensos entre los más jóvenes debido a que las restricciones a la movilidad por el Covid-19 han impactado negativamente en el sector de hostelería con una pérdida anual del $75 \%$ de afiliados a la seguridad social. Dicho sector emplea un gran número de trabajadores jóvenes de poca formación y que ahora requerirán una nueva adaptación (gráfico 2).

De este modo, en el año de la pandemia, a pesar de que los jóvenes representan el $4,4 \%$ del conjunto total del empleo han perdido 200.000 puestos de trabajo, 
equivalente al 32\% del empleo total, mientras que el número de personas ocupadas mayores de 44 años ha aumentado en 132.000.

\section{Composición de la fuerza laboral por sectores}

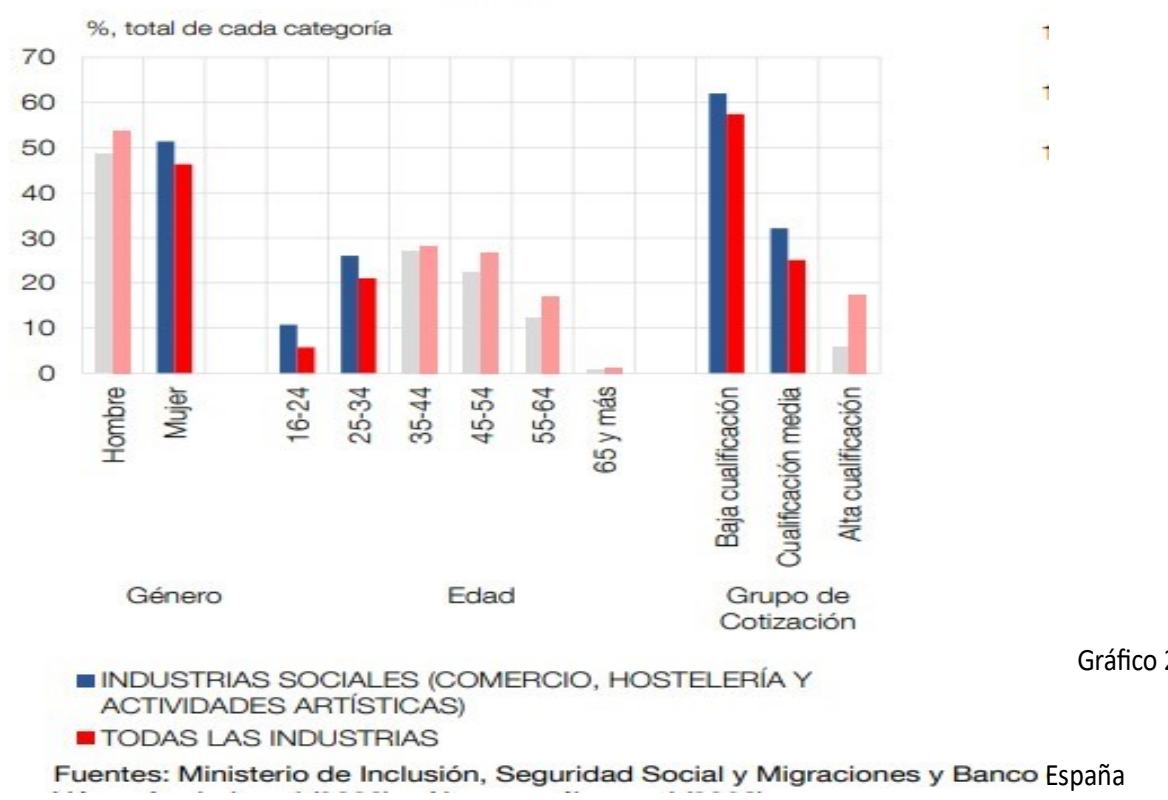

\section{DESIGUALDADES EN EL MERCADO LABORAL}

Y todo ello se produce en un mercado laboral con elevados desajustes: elevada tasa de desempleo juvenil, la más alta de la UE (tasa de paro del 40,13\%, tres veces más que la media de España) pero con una mayor incidencia entre los que no tienen estudios superiores (gráfico 3).

\section{Tasas de empleo y paro en la zona euro \\ Tasa de paro la más alta de la UE}

1 TASA DE EMPLEO. MENOS DE 25 ANOOS (a)

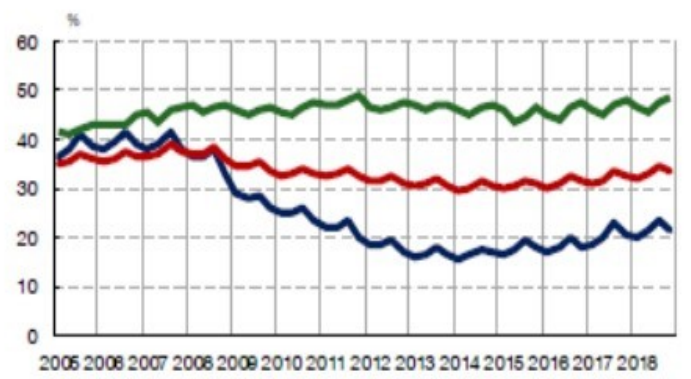

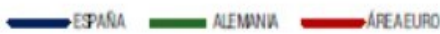

FUEITES: EUROSTAT y Banco de España.

a. En porcentaje de la población de ese grupo de edad.
2 TASA DE PARO. MENOS DE 25 AÑOS (a)

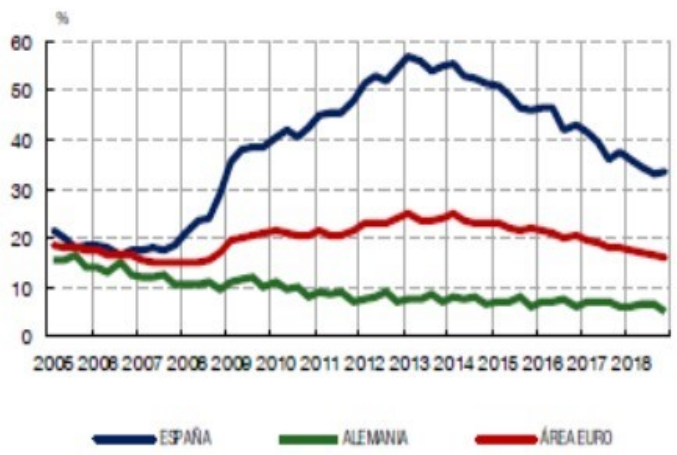

tasa de paro del $40,13 \%$ la más alta de la UE $(17,8 \%)$

Gráfico 3 
Son muchos los factores que han influido en que una vez más la destrucción de empleo se haya cebado entre los más vulnerables y que convierten el desempleo juvenil en un fenómeno estructural. Es el caso de aquellas personas que tienen un menor nivel de cualificaciones académicas y, por lo tanto, tienen una empleabilidad más reducida. Su formación es bastante desigual ya que el $21 \%$ de los parados jóvenes tienen estudios superiores, mientras el 79\% restante no los tienen (gráfico 4).

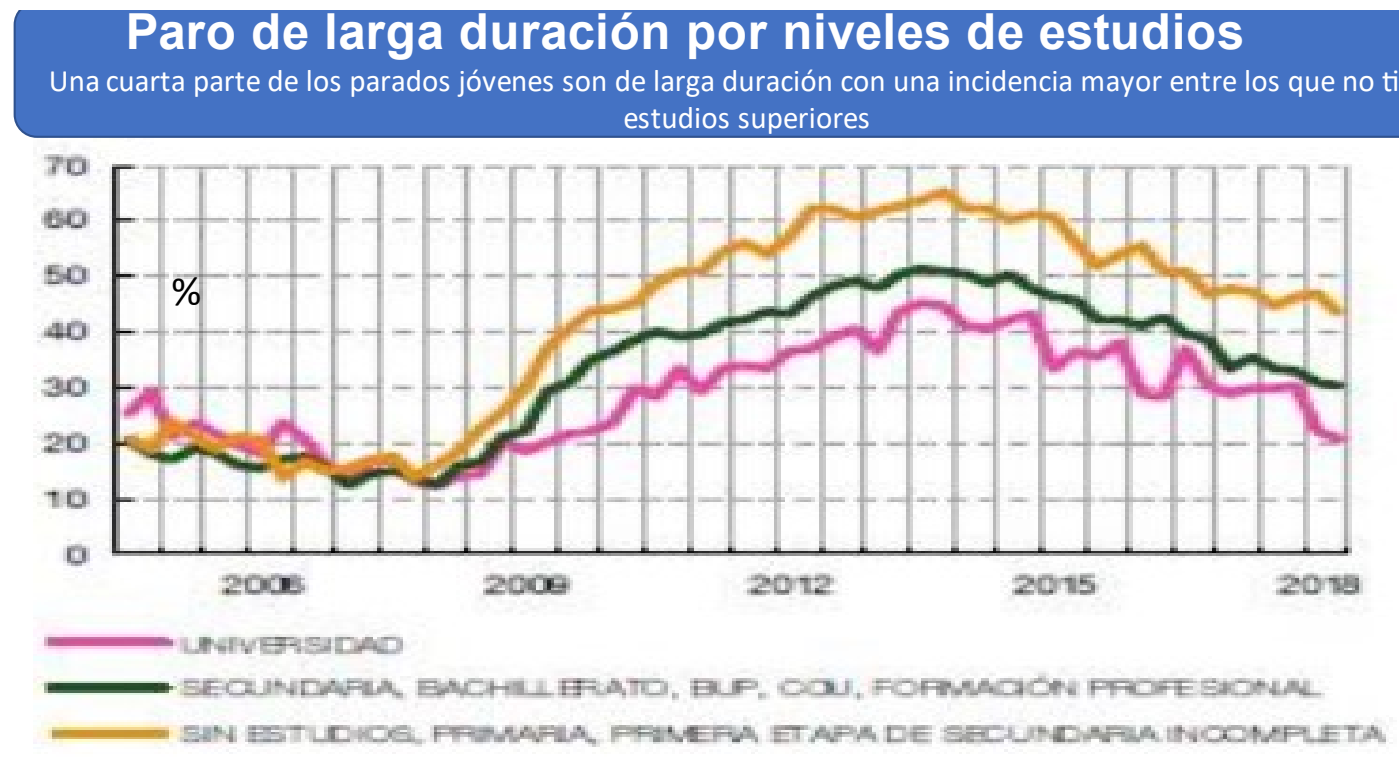

Fuente: INE y Bco. España

Gráfico 4

\section{CONDICIONES LABORALES MÁS PRECARIAS}

Otro factor se refiere a las condiciones laborales de los jóvenes españoles que son más precarias que en los países de nuestro entorno. Las entradas y salidas del mercado de trabajo se producen con contratos de trabajo temporales de corta duración y, en ocasiones, con excesiva sobrecualificación, lo que dificulta la acumulación de experiencia. Es decir, son los últimos en entrar al mercado de trabajo, pero son los primeros en salir cuando hay crisis por su mayor vulnerabilidad independientemente de su productividad. En concreto, el $67 \%$ tienen un contrato temporal (52\% en la zona euro) y un $36 \%$ tienen un contrato de jornada parcial (gráficos 5 y 6 ). 


\section{Las entradas y salidas del mercado de trabajo se producen con contratos temporales}

\section{ENTRADASAL EMPLEO CON NENOS DE 30 ANOOS (3)}

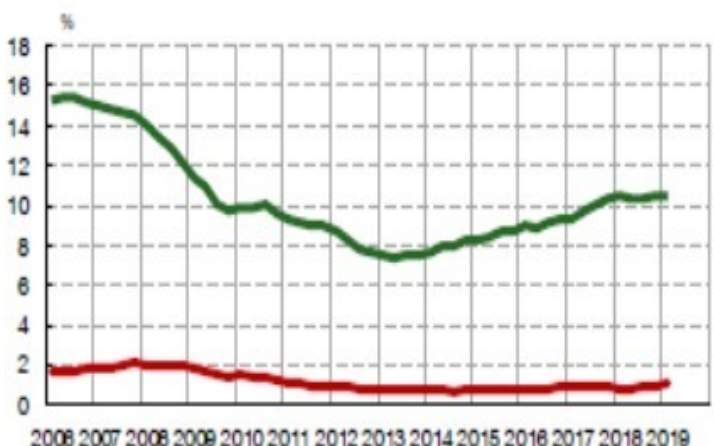

TIMPON NOENDO

PUENTES: Instituto Nacional de Estadistica y Banco de Epaf́a.
2 SAUDAS DEL EUPEO CON MENOS DE 30 ANOOS (a)

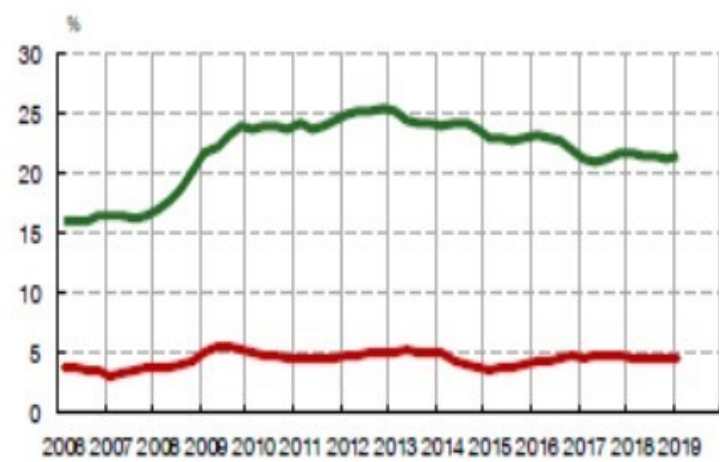

- TRMPON

MNERND

\section{TASA DE TEMPORALIDAD DE LOS JÓVENES (a)}

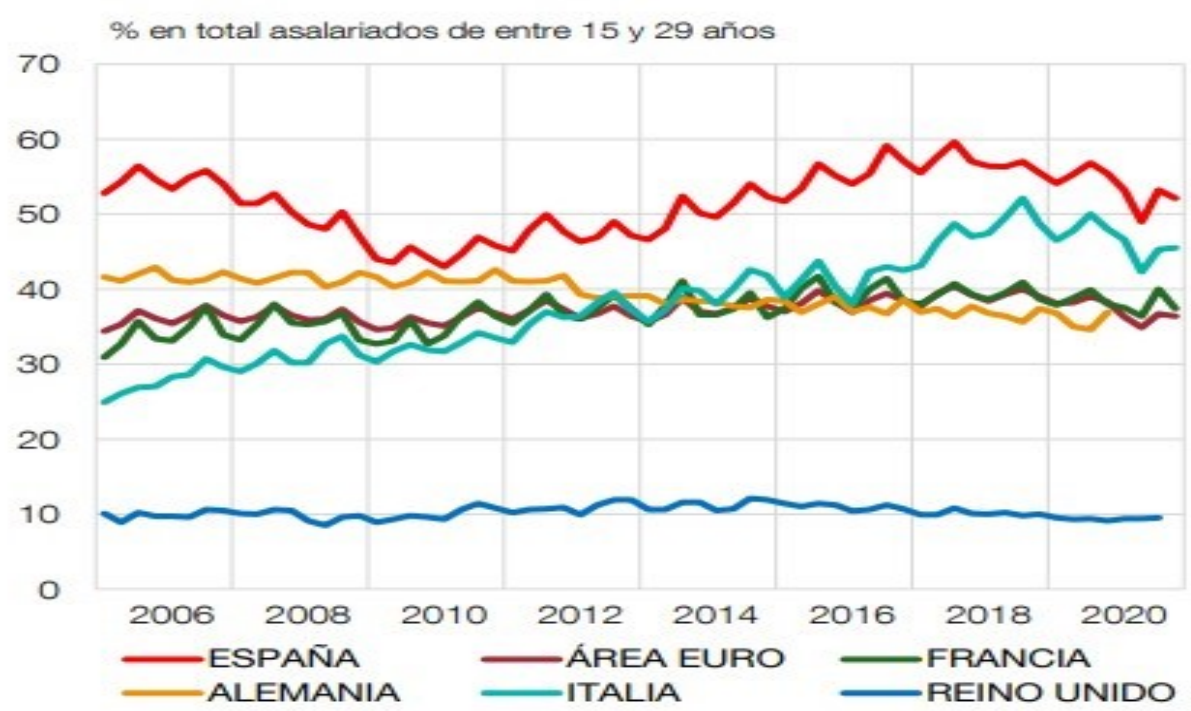

Fuente: Eurostat y Banco de España.

Gráfico 6

De esta forma, el paro juvenil en España se ha agravado durante la crisis sanitaria y económica derivada de la Covid-19: en el año de la pandemia se han perdido 200.000 puestos de trabajo, el 32\% del empleo total, pese a que los jóvenes representan el $4,4 \%$ del conjunto del empleo. 


\section{DIFICULTADES EN EL ACCESO A LA VIVIENDA}

El agravante de todo ello para los jóvenes se traduce en otro factor de enorme repercusión: las dificultades de acceso a la compra de una vivienda. La elevada inestabilidad de los contratos temporales y parciales limita la capacidad de obtener ingresos estables periódicamente. A ello, habría que añadir el endurecimiento de la oferta de crédito. Es complicado, por no decir imposible, obtener una hipoteca con contratos de trabajo de corta duración.

Según el Banco de España, la proporción de individuos jóvenes que poseen su vivienda de residencia a los 30 años ha ido disminuyendo para los nacidos a partir de 1980: el 7\% de los nacidos en 1988 poseía su vivienda principal a los 29 años; entre los nacidos en 1976, ese porcentaje era del 26\% (gráfico 6).

España tiene una de las edades de emancipación más tardías de Europa. Se emancipan, como media, a los 29,8 años mientras la edad media de emancipación en la UE-27 es de 26,2 años, según Eurostat, (abril 2020). Además, en España el $64 \%$ de las personas de 25 a 29 viven con sus padres, una cifra bastante superior a la UE (42\%) y a países de nuestro entorno como Francia (17\%), Reino Unido (25\%) o Alemania (30\%). Ello, es debido a diversos factores entre los que hay que destacar: el desempleo, la precariedad y los insuficientes niveles salariales. En concreto, dedican un 51\% de su renta salarial al pago de alquiler cuando se aconseja dedicar un 30\% al pago de la vivienda (Instituto de la Juventud, 2021).

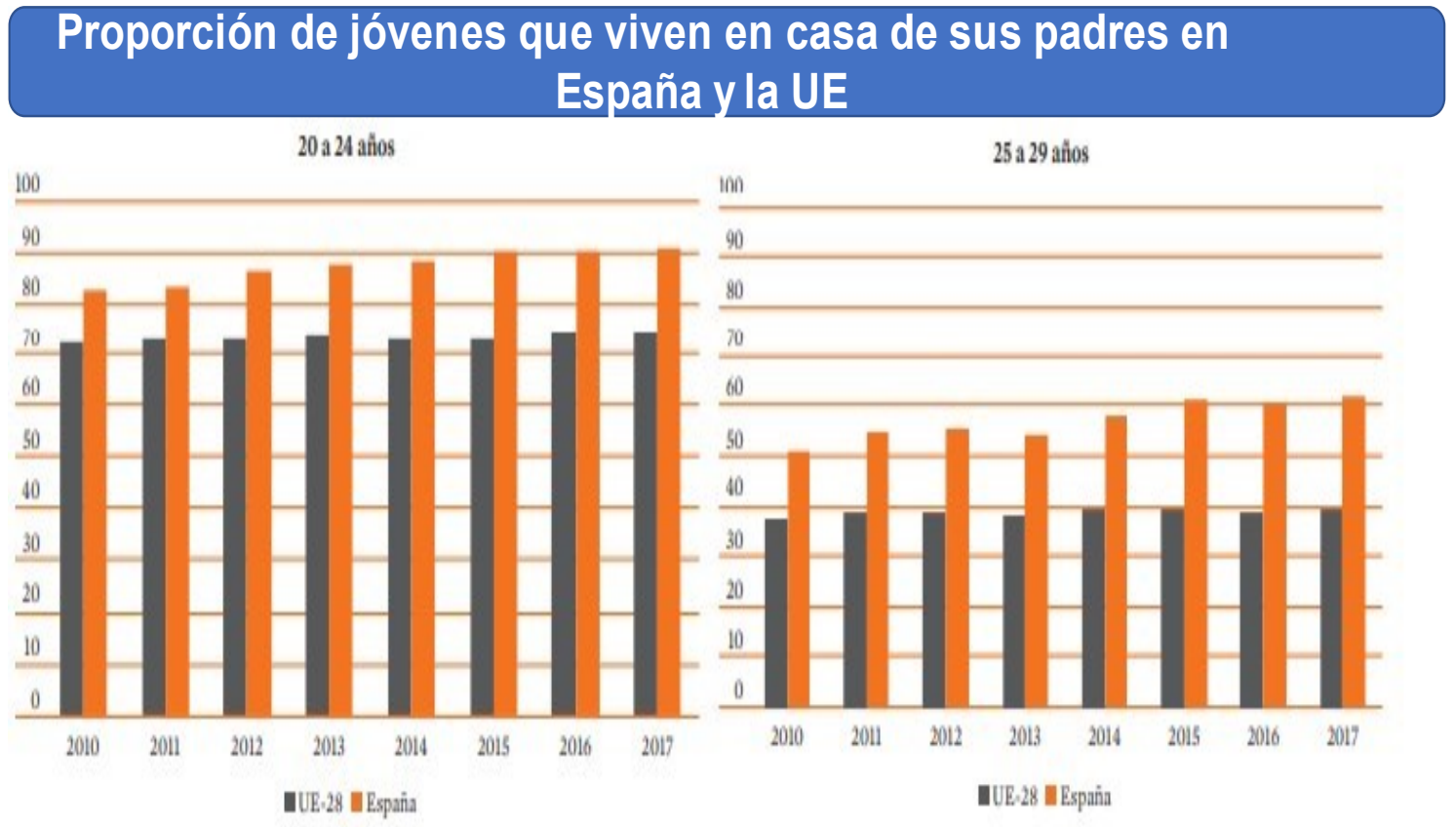

Fuente: Eurostat, Population and Social Conditions, 2019.

Gráfico 6 


\section{ANTE UN PROBLEMA SISTÉMICO, SOLUCIONES MÚLTIPLES}

Debido a que nos encontramos ante un problema sistémico las soluciones deberán ser múltiples. Entre ellas, la renovación del sistema educativo y el impulso de las Políticas Activas de empleo.

\subsection{RENOVACIÓN SISTEMA EDUCATIVO}

Nuestro entorno muta y nuestras habilidades deberían hacerlo al mismo ritmo. Por ello, es necesario adaptar nuestra educación a la tecnología actual y futura como estrategia de relación permanente con el entorno para abordar riesgos y aprovechar oportunidades. Así, es fundamental la educación aplicada a las necesidades de la empresa y la gestión del talento para favorecer la empleabilidad.

Por todo ello, y como respuesta para aminorar el problema se debe actuar conjuntamente en los ámbitos educativo y laboral mediante la adopción de reformas y cuya prioridad debe ser disminuir primeramente la tasa de paro del conjunto de la población activa. Por lo tanto, el paro de los jóvenes no es solamente un problema económico, es también un problema político y, como tal, debe tratarse.

El cambio del sistema educativo es imprescindible. El modelo educativo actual necesita una revisión. Por ello, hay que modernizar el sistema de aprendizaje y el contenido del currículum educativo, para dotar a los estudiantes de herramientas con las que enfrentarse a un entorno más tecnológico y cambiante.

La formación profesional (FP) por su papel estratégico requiere una revisión profunda que permita aumentar y adecuar el conocimiento y habilidades a lo largo de la vida, para lo que es fundamental ajustar la estructura formativa a las necesidades del sistema productivo.

En este contexto, hay que mejorar, priorizar y dignificar la FP ya que proporciona rutas más directas al mercado laboral y es más flexible por su adaptación (tasa de paro $8 \%$, frente al $40 \%$ de los jóvenes). Sólo el 19\% del alumnado de 17 años elige la FP frente a la media de la OCDE del 31,0\%. Según el Observatorio de las Ocupaciones del SEPE de 2020, la tasa de empleabilidad de esta modalidad de formación llegó al 42,2\%. De este modo, estas serían las sugerencias más importantes:

- Formación en competencias fundamentales (lenguaje, matemáticas, ciencias y competencia global). La OCDE: las mejores expectativas de empleo están en aquellos alumnos que tengan habilidades en el campo de las tecnologías de la información y la comunicación (TIC).

- Formación en habilidades transferibles (pensamiento crítico, solución de problemas, comunicación, gestión de conflictos, trabajo en equipo, etc.).

- Promover competencias digitales 
Los jóvenes españoles siguen mostrando unos niveles de competencias básicas por debajo de los de la mayoría de los países desarrollados y de nuestros homólogos europeos. Además, los bajos niveles de competencias se mantienen en todos los estadios educativos, incluso en la universidad, según un informe del Consejo Económico y Social: Jóvenes y mercado de trabajo en España.

Recientemente el Foro Económico Mundial (en sus siglas en ingles WEF) indicaba que las habilidades estaban cambiando y destacaba aquellas que consideraba entre las más importantes: pensamiento analítico y de innovación, aprendizaje activo y estratégico, resolución de problemas complejos (gráfico 7).

\section{Las 12 habilidades que serán más demandadas \\ hasta el 2025 (creatividad, razonamiento, comunicación, trabajo en equipo, negociación)}

1. Pensamiento analítico y de innovación

2. Aprendizaje activo y estratégico

3. Resolución de problemas complejos

4. Pensamiento crítico

5. Creatividad, originalidad e iniciativa

6. Liderazgo e influencia social

7. Uso de la tecnología

8. Diseño y programación de tecnología

9. Resiliencia, tolerancia al estrés y flexibilidad

10.Razonamiento y resolución de problemas

11.Inteligencia emocional

12.Persuasión y negociación
Gráfico 7

Fuente: elaboración propia en base a datos WEF

\subsection{IMPULSAR POLÍTICAS ACTIVAS DE EMPLEO}

Es conveniente impulsar el gasto de las Políticas Activas de Empleo en actividades de formación y de orientación, evaluar la eficiencia y productividad de las medidas de inserción, formación de trabajadores, incentivos para el sector privado y servicios de orientación y seguimiento (gráfico 8). 


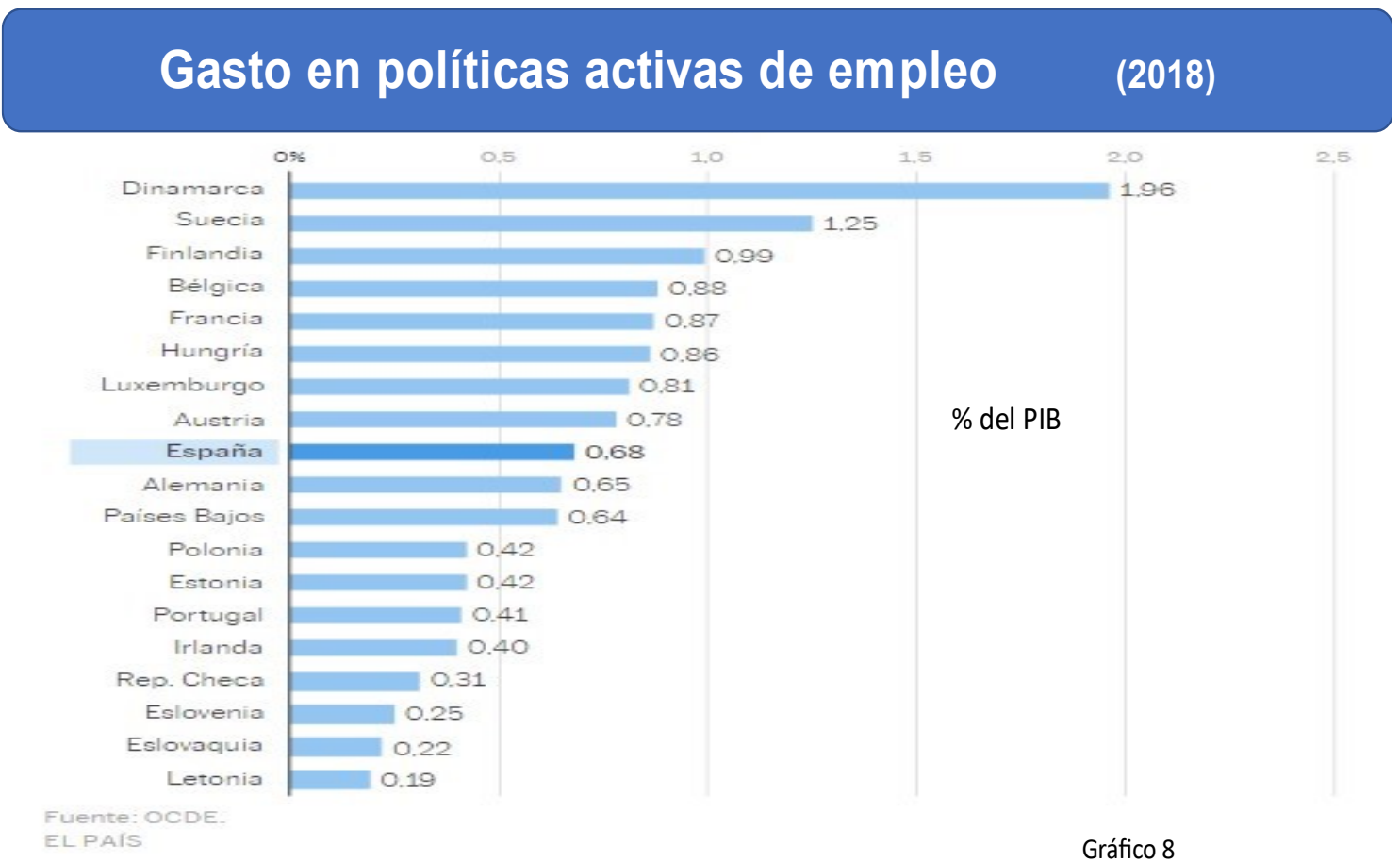

Para afrontar esta situación es una buena señal que el Gobierno haya aprobado recientemente Plan de Garantía Juvenil Plus (2021-2027), una iniciativa de la Comisión Europea para luchar contra el paro juvenil. Su dotación es de 3.263 millones de euros, que se incluye dentro de la Estrategia Juventud Avanza con una inversión total de casi 5.000 millones de euros.

Es importante que los fondos se destinen a Políticas Activas de Empleo. Según la OCDE, un aumento del $10 \%$ del stock de capital humano acumulado a través de la formación en el puesto de trabajo, (on-the-job training), se traduce en un aumento del orden de 1,5 puntos porcentuales del nivel de productividad. Ello facilita la asignación de recursos humanos hacia actividades con productividades superiores a la media, mejorando la adecuación entre ofertas y demandas de empleo y favoreciendo los cambios tecnológicos. En contrapartida, las políticas activas que incrementan el empleo, pero aumentando la proporción de trabajadores de baja cualificación que, paralelamente, frenan el crecimiento de la productividad y, por esta razón, tienen efectos globalmente perversos sobre el crecimiento económico y nivel de vida.

El Banco de España, en un informe reciente, concluye: muchos de los retos de la economía española anteriores a la pandemia, que con frecuencia se han visto agudizados por esta, afectan de manera especial a los jóvenes: sistema educativo, mercado de trabajo, vivienda, incertidumbre e inestabilidad de la renta...

La sociedad en su conjunto no puede permitir que los más jóvenes se vean obligados a emigrar y que sean los más castigados por cualquier tipo de crisis económica o pandémica. Es una exigencia preocuparnos especialmente por la situación de los jóvenes. No podemos resignarnos ante la difícil situación que afrontan las próximas generaciones. Sin olvidar que es una prioridad absoluta, no solo por el bien de los jóvenes, sino del conjunto de la sociedad. Los daños producidos en el mercado laboral, amplificados por el COVID-19, no se repararán fácilmente. 


\section{REFERENCIAS}

- Banco de España (2021). La crisis del covid-19 y su impacto sobre las condiciones económicas de las generaciones jóvenes. Madrid.

- Banco de España (2019). La juventud en España: retos y oportunidades. Madrid

- Consejo Económico y Social de España (2020). Jóvenes y mercado de Trabajo en España. Madrid.

- Comisión Europea. Employment and Social Developments in Europe. Annual Review 2017.

- Comisión Europea: Empleo Juvenil. Review. 09/2017.

- Christian Vulliez (2013). Les jeunes et l'emploi. Groupe Eyrolles. Paris

- Instituto de la Juventud (2021). Resumen ejecutivo informe juventud en España 2020. Madrid. 